\title{
Non-homogeneous thermal boundary conditions in low Prandtl number pipe flows
}

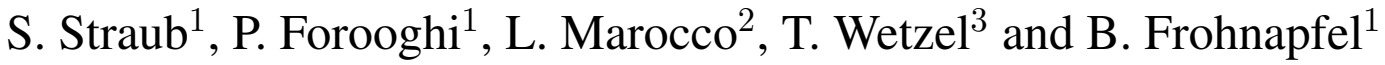 \\ ${ }^{1}$ Institute of Fluid Mechanics, Karlsruhe Institute of Technology, \\ Kaiserstr. 10, 76131 Karlsruhe, Germany. steffen.straub@kit.edu \\ ${ }^{2}$ Dipartimento di Energia, Politecnico di Milano, \\ Via Lambruschini 4, 20156 Milano, Italy. \\ ${ }^{3}$ Institute of Thermal Process Engineering, Karlsruhe Institue of Technology, \\ Kaiserstr. 12, 76131 Karlsruhe, Germany.
}

\begin{abstract}
The effect of non-homogeneous thermal boundary conditions on temperature statistics in low Prandtl number turbulent pipe flows is studied numerically via direct numerical simulations. Two wall heat flux distributions, varying in azimuthal direction and motivated by concentrated solar power systems, are prescribed and their influence on the thermal field is presented. As a reference, also homogeneous thermal boundary conditions are simulated and compared the the non-homogeneous ones. The influence of the azimuthal variation of prescribed wall heat flux is assessed in terms of instantaneous velocity and temperature fields, local and global Nusselt numbers, averaged temperature distributions and the turbulent thermal diffusivity. The global Nusselt number appears to be unaffected by the thermal boundary conditions, whereas the local Nusselt number deviates appreciably.
\end{abstract}

\section{Introduction}

The large-scale application of profitable, renewable energy is one of the major challenges for our generation. One promising technique among concentrating solar power systems are central receiver systems, which focus solar radiation onto a receiver where it is transferred to a coolant fluid. Recently, liquid metals have been proposed as heat transfer fluid due to their high thermal conductivity [1]. In the present numerical simulation only a single pipe of a receiver is considered for the simulations. There are only a few published direct numerical simulation (DNS) datasets on heat transfer in turbulent pipe flows, typically at low Reynolds numbers, e.g. Ref. $[2,3]$. More recently, numerical simulations also tackle non-homogeneous thermal boundary conditions [4-8]. Nevertheless, there is still a lack of openly available high-fidelity datasets for heat transfer in turbulent pipe flows, especially concerning low Prandtl number fluids.

Therefore, the first objective of the present study is to fill this gap for homogeneous and non-homogeneous boundary conditions by creating a database of high fidelity DNS results. Such a dataset is valuable for improving Reynolds-Averaged-Navier-Stokes (RANS) models to properly capture the effects of low Prandtl numbers and non-homogeneous thermal boundary conditions. Second objective, more directly related to the engineering problems in central receiver systems, is to provide information about the temperature statistics at the fluid - solid interface. Due to the inhomogeneous distribution of the temperature around the receiver tube, arising thermal stresses need to be considered during the design of the receiver [9-11]. Hence, accurate results of the temperature distribution are necessary. Finally, a better understanding of the effects of spatially inhomogeneous thermal boundary conditions on the heat transfer mechanisms, especially for low Prandtl number fluids, is sought.

The paper is structured as follows. Numerical methods and definition of the investigated thermal boundary conditions is given in the next section. Section 3 presents results in terms of instantaneous velocity and temperature fields, global and local Nusselt numbers, averaged 
temperature distributions, and the turbulent thermal diffusivity as well as the turbulent Prandtl number. Finally, the main findings are summarized in section 4.

\section{Numerical Methods}

The highly parallelized spectral elements code Nek5000 [12] is employed for numerically solving the governing equations partial differential equations for velocity and temperature. In the spectral element method, originally proposed by Patera [13], the domain is divided into smaller elements. Within each element, the unknowns velocity $\boldsymbol{U}$, pressure $p$, and temperature $T$ are represented as Lagrangian interpolants based on Gauss-Lobatto-Legendre quadrature nodes. The polynomial order of the Lagrangian interpolants is set to $N=7$ for velocity and temperature and $N=5$ for pressure, so called $\mathbb{P}_{N}-\mathbb{P}_{N-2}$ formulation. Material properties are assumed constant, buoyancy effects are neglected, and a fully developed state is considered.

The domain size is defined by the pipe length as $L_{z}=12.5 D$, where $D=2 R$ is the diameter, according to previous studies $[5,14,15]$. Radial, azimuthal and streamwise coordinates are denoted by $r, \varphi$ and $z$, respectively. The mesh is constructed such that $\Delta r^{+}<5,(\Delta R \varphi)^{+}<5$, $\Delta z^{+}<10$, the first grid point away from the wall is located at $y^{+} \approx 0.15$ and fourteen grid points are below $y^{+}=10$. The bulk Reynolds number is prescribed at $R_{b}=U_{b} D / \nu=5300$, with $U_{b}$ and $\nu$ denoting the bulk velocity and kinematic viscosity, respectively. Two Prandtl numbers, $\operatorname{Pr}=0.71$ and $\operatorname{Pr}=0.025$, are considered. Time averaging is over $950 \mathrm{D} / U_{b}$ corresponding to 76 flow-throughs of the domain and the CFL Number is kept around 0.35.

Investigated thermal boundary conditions are non-homogeneous in azimuthal direction, permitting periodic boundary conditions at inlet and outlet when solving for a non-dimensionalized temperature $\Theta$ instead of $T$.

$$
\Theta(r, \varphi, z, t)=\frac{\left\langle T_{b}\right\rangle^{t}(z)-T(r, \varphi, z, t)}{T_{r e f}}, \quad T_{b}=\frac{\int_{A} U_{z} T d A}{A U_{b}}, \quad T_{r e f}=\frac{\left\langle q_{w}\right\rangle^{\varphi, z, t}}{\rho c_{p} U_{b}}
$$

such that $d\langle\Theta\rangle^{t} / d z=0$. Here, the operator \langle\rangle with superscripts $r, \varphi, z, t$ denotes averages over the according spatial direction or time. Besides, $\left\langle T_{b}\right\rangle^{t}$ is the time-averaged bulk temperature, $A$ the cross section of the pipe, $T_{\text {ref }}$ a reference temperature composed of mean wall heat flux $\left\langle q_{w}\right\rangle^{\varphi, z, t}$, density $\rho$, specific heat capacity $c_{p}$, and bulk velocity $U_{b}$.

The simulation is run at constant flow rate with the prescribed bulk Reynolds number and the governing equations being solved by the code are the Navier-Stokes equations and an advection-diffusion equation for $\Theta$ (both scaled by $U_{b}$ and $D$ ).

$$
\begin{aligned}
\frac{\partial \boldsymbol{U}}{\partial t}+(\boldsymbol{U} \cdot \nabla) \boldsymbol{U} & =-\nabla p+\frac{1}{R e_{b}} \nabla^{2} \boldsymbol{U}+\boldsymbol{f}, \quad \nabla \cdot \boldsymbol{U}=0, \\
\frac{\partial \Theta}{\partial t}+\boldsymbol{U} \cdot \nabla \Theta & =\frac{1}{P e} \nabla^{2} \Theta+q_{v o l},
\end{aligned}
$$

with a forcing term $\boldsymbol{f}$ imposing the constant flow rate, the Péclet number $P e=U_{b} D / \alpha$, where $\alpha=k /\left(\rho c_{p}\right)$ is the thermal diffusivity and $k$ is the thermal conductivity, and a volumetric source term $q_{v o l}$. The boundary conditions for the velocity field are no-slip at the wall and periodic at inlet and outlet. For the temperature field, two different distributions of wall heat flux are considered:

$$
\begin{aligned}
& \text { halfconst: } \quad q_{w}(\varphi)=2\left\langle q_{w}\right\rangle^{\varphi, z, t}, \quad 0<\varphi<\pi, \quad q_{w}=0, \quad \text { else, } \\
& \text { halfsin: } \quad q_{w}(\varphi)=\pi\left\langle q_{w}\right\rangle^{\varphi, z, t} \sin (\varphi), \quad 0<\varphi<\pi, \quad q_{w}=0, \quad \text { else, }
\end{aligned}
$$


i.e. only half of the tube's surface is kept adiabatic and the other half is prescribed with a constant or sinusoidal wall heat flux distribution. In both cases the azimuthally averaged wall heat flux is equal to the homogeneous reference cases. To compensate for the linear increase of $\left\langle T_{b}\right\rangle^{t}$ in streamwise direction (see e.g. Ref. [16]), the volumetric source term is $q_{v o l}=4 U_{z} / U_{b}$.

Three different types of homogeneous boundary conditions are investigated additionally: ideal isoflux (IFi) with a prescribed wall heat flux constant over time and space, ideal isothermal (IT) with a wall temperature constant over time and space, and a mixed-type (IFm) boundary condition, where the time-averaged wall heat flux is constant in space and the wall temperature is constant in time. For details of the governing equations and boundary conditions of the homogeneous setups see Piller [2].

\section{Results}

This section is divided into four subsections presenting instantaneous velocity and temperature fields, global and local Nusselt numbers, averaged temperature fields, and turbulent thermal diffusivity as well as turbulent Prandtl number. Note that the results for the averaged temperature are reported in viscous units (denoted by a superscript ${ }^{+}$), i.e. the friction velocity $u_{\tau}=\sqrt{\tau_{w} / \rho}$, where $\tau_{w}$ denotes the wall shear stress, and $\nu$ are used instead of $U_{b}$ and $D$ for non-dimensionalization.

\subsection{Instantaneous velocity and temperature fields}

The ideal isoflux boundary condition for the homogeneous setup as well as both inhomogeneous setups only prescribe the wall heat flux, thereby permitting temperature to fluctuate at the pipe wall. This is visualized in Fig. 1 for the instantaneous velocity and non-dimensionalized temperature at the same instant in time of the homogeneous IFi setup. For the velocity field, streaky structures of low-speed and high-speed velocity can be observed close to the wall at a radial location of $r=0.48 D$. Thermal structures right at the wall at $r=0.5 D$ show features very similar to the velocity field for the $\operatorname{Pr}=0.71$ case. In regions of low-speed velocity (dark blue), also the non-dimensionalized temperature of the homogeneous IFi setup $\Theta_{h}$ is low (in fact, negative). As $\Theta_{h}$, in the IFi setup, is defined as $\Theta_{h}=\left(\left\langle T_{w}\right\rangle^{\varphi, t}-T\right) / T_{\text {ref }}$, low values of $\Theta_{h}$ (i.e. negative values of high magnitude) correspond to instantaneous temperatures higher than the averaged wall temperature.

In the non-homogeneous setups, the non-dimensionalized temperature field $\Theta$, as defined above, varies mostly in the upper part of the pipe, shown in Fig. 2. For halfsin071 (top), the gradients in the temperature field are higher than for halfsin0025 (bottom) due to the high thermal conductivity which tends to reduce the temperature gradients. Note that the scaling is

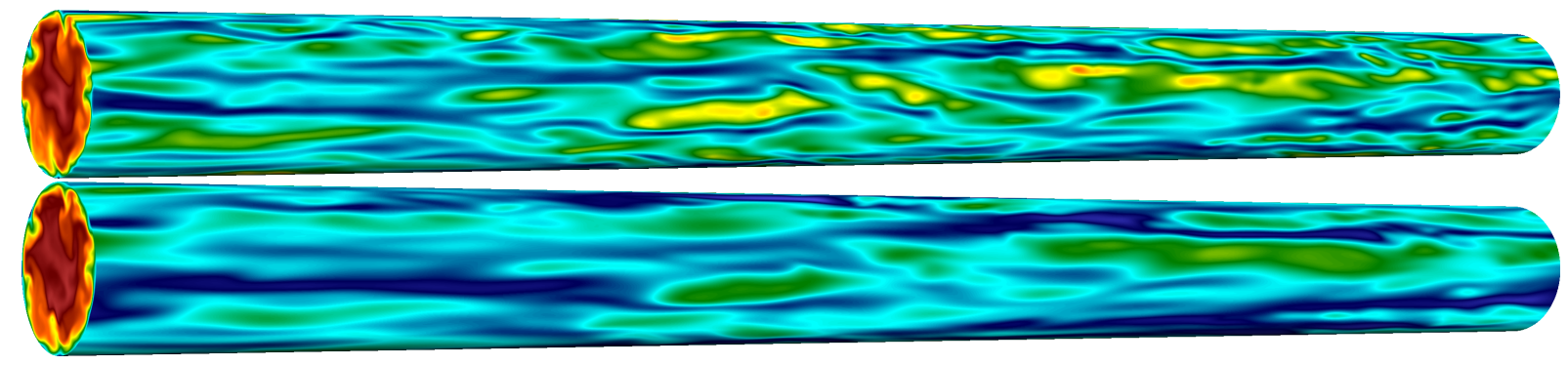

Figure 1: Instantaneous snapshots of (top) velocity magnitude at $r=0.48 D$ and (bottom) non-dimensionalized temperature field of IF-i at $\operatorname{Pr}=0.71$ at $r=0.5 D$. 


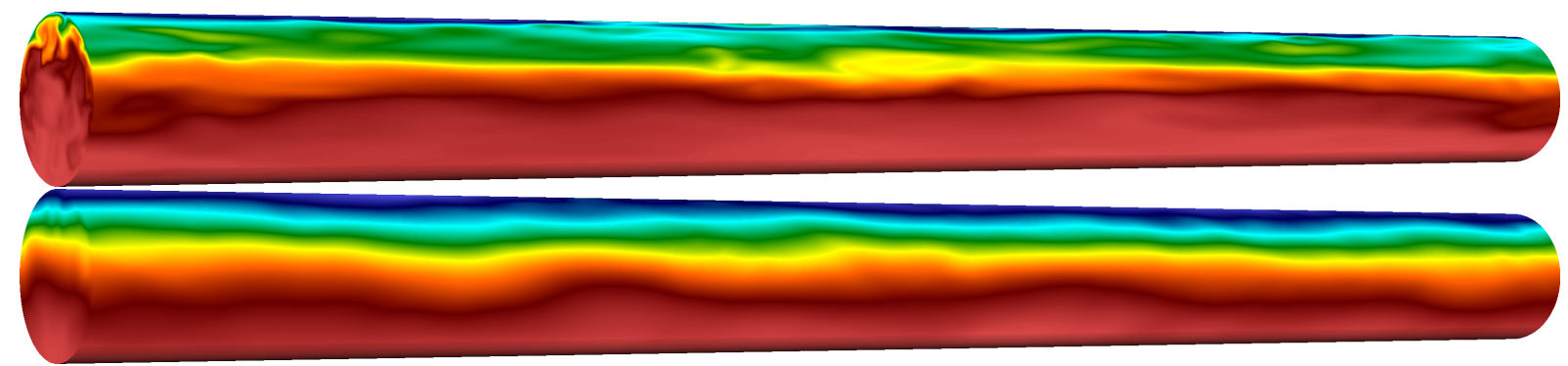

Figure 2: Instantaneous snapshots of non dimensionalized temperature field of halfsin at (top) $\operatorname{Pr}=0.71$ and (bottom) $\operatorname{Pr}=0.025$.

different for both snapshots.

As instantaneous results are only of limited value in turbulence research, statistically averaged quantities, which are reproducible, need to be considered. This is done in the next subsections.

\subsection{Nusselt number}

An important quantity in turbulent heat transfer is the Nusselt number $N u=h D / k$, where $h=q_{w} /\left(T_{w}-T_{b}\right)$ is the heat transfer coefficient. We define three Nusselt numbers for the azimuthally inhomogeneous setups as

$$
\begin{aligned}
N u^{G} & =\frac{D}{k} \frac{\left\langle q_{w}\right\rangle^{\varphi, z, t}}{\left\langle T_{w}\right\rangle^{\varphi, z, t}-\left\langle T_{b}\right\rangle^{z, t}}, \\
N u(\varphi) & =\frac{D}{k} \frac{q_{w}(\varphi)}{\left\langle T_{w}\right\rangle^{z, t}(\varphi)-\left\langle T_{b}\right\rangle^{z, t}}, \\
\langle N u\rangle^{\varphi} & =\frac{D}{k}\left\langle\frac{q_{w}(\varphi)}{\left\langle T_{w}\right\rangle^{z, t}(\varphi)-\left\langle T_{b}\right\rangle^{z, t}}\right\rangle^{\varphi} .
\end{aligned}
$$

$N u^{G}$ is a global Nusselt number using the averaged wall heat flux $\left\langle q_{w}\right\rangle^{\varphi, z, t}$. It is related to $\Theta$ by $N u^{G}=-P e /\left\langle\Theta_{w}\right\rangle^{\varphi, z, t}$. Analogously, a local Nusselt number $N u(\varphi)$, taking into account the azimuthal variation of $q_{w}$ and related to $\Theta$ by $N u(\varphi)=-P e /\left\langle\Theta_{w}\right\rangle^{z, t} \cdot q_{w}(\varphi) /\left\langle q_{w}\right\rangle^{\varphi, z, t}$ is defined. Finally, averaging the local Nusselt number in azimuthal direction yields $\langle N u\rangle^{\varphi}$.

They are compared in Table 1 for the investigated azimuthal distributions of wall heat flux. Evidently, the global Nusselt number $N u^{G}$ and the averaged Nusselt number $\langle N u\rangle^{\varphi}$ are strongly affected by the Prandtl number. However, the distribution of wall heat flux, being halfconst or halfsin, appears to be negligible for $N u^{G}$ and only plays a role for $\langle N u\rangle^{\varphi}$. Moreover, the averaged Nusselt number $\langle N u\rangle^{\varphi}$ is significantly lower than the global $N u^{G}$, explained by the distribution of $\left\langle\Theta_{w}\right\rangle^{z, t}$ (see section 3.3.). In short, at azimuthal locations of large wall heat

Table 1: Nusselt numbers for varying distributions of wall heat flux.

\begin{tabular}{ccccc}
\hline & halfconst071 & halfsin071 & halfconst0025 & halfsin0025 \\
\hline$N u^{G}$ & 18.44 & 18.53 & 6.45 & 6.49 \\
$\langle N u\rangle^{\varphi}$ & 7.81 & 7.42 & 2.01 & 1.63 \\
\hline
\end{tabular}


Table 2: Global Nusselt numbers for varying types of thermal boundary conditions.

\begin{tabular}{ccccccc}
\hline & IT071 & IFm071 & IFi071 & IT0025 & IFm0025 & IFi0025 \\
\hline present & 17.56 & 18.29 & 18.39 & 5.10 & 6.42 & 6.45 \\
Piller [2] & 17.44 & 18.54 & 18.32 & - & - & - \\
\hline
\end{tabular}

flux $q_{w}(\varphi)$ also the temperature difference $\left\langle T_{w}\right\rangle^{z, t}(\varphi)-\left\langle T_{b}\right\rangle^{z, t}$ is large and at locations of small temperature difference, the wall heat flux vanishes.

Note that, for the homogeneous reference cases, the global Nusselt number $N u^{G}$ and the averaged Nusselt number $\langle N u\rangle^{\varphi}$ are identical by definition. Thus, only the global Nusselt number for the three homogeneous setups is presented in Table 2. First, the global Nusselt numbers for both homogeneous isoflux cases, IFm and IFi, and the azimuthally non homogeneous setups differ at most by $1.3 \%$ at the same Prandtl number. Second, good agreement between the present results and Piller [2] is found. Third, the isothermal boundary conditions yield lower Nusselt numbers [2], due to the higher bulk temperature, also for the low Prandtl number setups. Finally, comparing the present results to existing correlations, good agreement is found for $\operatorname{Pr}=0.71$ where the correlation by by Gnielinski [17] yields $N u_{\text {Gniel }}=17.34$. For the lower Prandtl number of $\mathrm{Pr}=0.025$ two correlations are selected according to Pacio, Marocco, and Wetzel [18]. The correlation by Lubarsky and Kaufman [19] yields $N u_{L u b}=4.41$ and the one by Skupinski, Tortel, and Vautrey [20] yields $N u_{S k u p}=4.85$, both considerably lower than the present simulation results. One reason for this discrepancy could be the negligence of buoyancy forces, i.e. forced convection, in the present simulation, whereas at this low Reynolds number the experiments might be considerably influenced by buoyancy forces, i.e. mixed convection.

The local Nusselt number for the azimuthally inhomogeneous setups is shown in Fig. 3 for $0 \leq \varphi \leq \pi / 2$ as the problem is symmetric around $\varphi=\pi / 2$ and the local Nusselt number vanishes on the lower side of the pipe. A maximum for all local Nusselt numbers is found at $\varphi \approx 0$. Due to the smoother transition of halfsin071 the peak is not as strongly pronounced as for halfconst071. In the case of halfsin0025, the temperature difference in the denominator of eq. (7) vanishes for $\varphi \gtrsim 0$ resulting in a singularity, whereas for all the other cases the denominator in eq. (7) vanishes for $\varphi<0$. Furthermore, the local Nusselt number at the
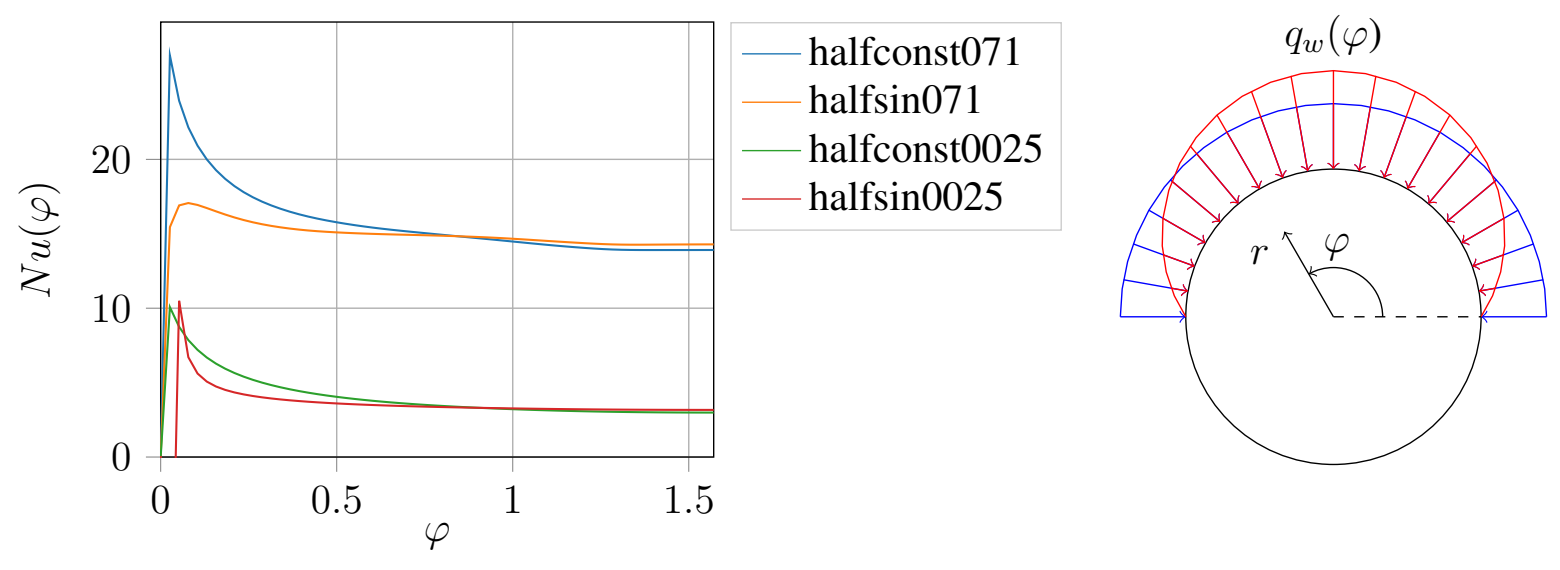

Figure 3: Local Nusselt number distribution and sketch of coordinate system. 

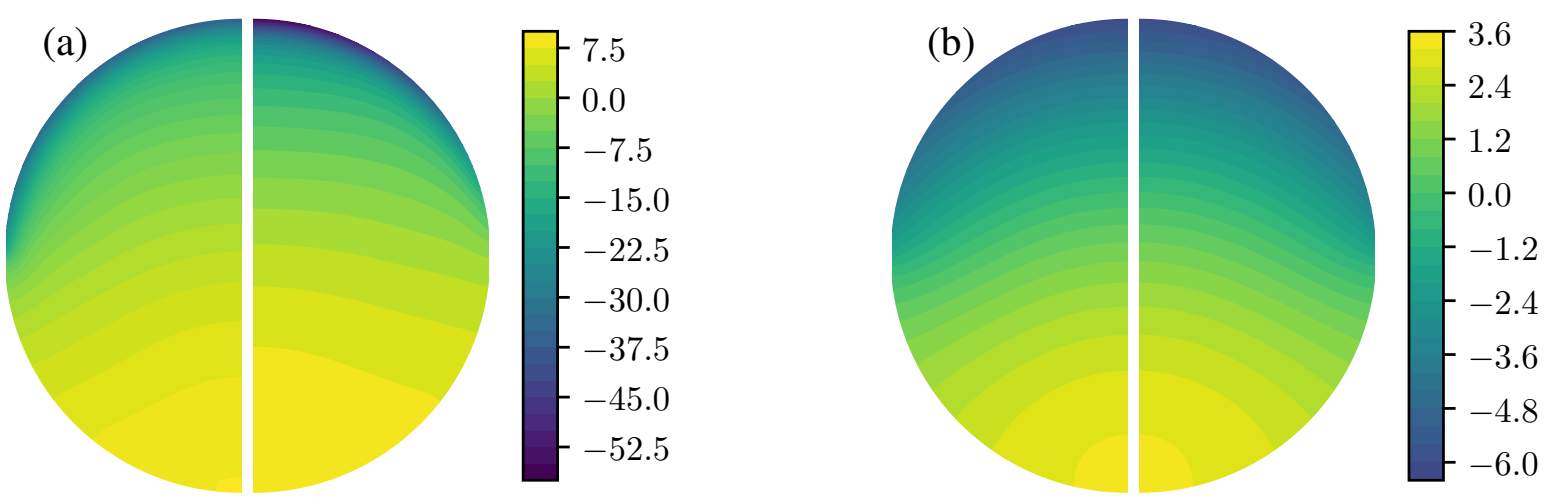

Figure 4: Non-dimensionalized temperature $\left\langle\Theta^{+}\right\rangle^{z, t}$ for (a) $\operatorname{Pr}=0.71$ and (b) $\operatorname{Pr}=0.025$ with halfconst on the right and halfsin on the left side of each plot.

angle of $\varphi=\pi / 2$ reaches values considerably smaller than $N u^{G}$ of $13.91,14.29,2.99,3.17$ for halfconst071, halfsin071, halfconst0025, and halfsin0025, respectively.

In order to understand the local Nusselt number behaviour better, the difference between bulk temperature and wall temperature needs to be evaluated. Thus, the distribution of the averaged non-dimensionalized temperature as well as the averaged non-dimensionalized wall temperature is shown in the next subsection.

\subsection{Temperature statistics}

2D contour plots of the non-dimensionalized temperature field $\left\langle\Theta^{+}\right\rangle^{z, t}$ are given in Fig. 4. Left-right symmetry has been exploited to improve the statistics. The difference in $q_{w}(\varphi)$ can be observed in Fig. 4 (a) with a dark blue region for halfsin071 at $\varphi=\pi / 2$, whereas for halfconst $\left\langle\Theta^{+}\right\rangle^{z, t}$ is more evenly distributed around the wall. For the lower Prandtl number, halfconst and halfsin are barely distinguishable and much smoother temperature gradients are observed due to the high molecular conductivity.

In order to compare the effects of $q_{w}(\varphi)$ more quantitatively, Fig. 5 presents profiles of the non-dimensionalized wall temperatures $\left\langle\Theta_{w}^{+}\right\rangle^{z, t}$. This behaviour explains the small local
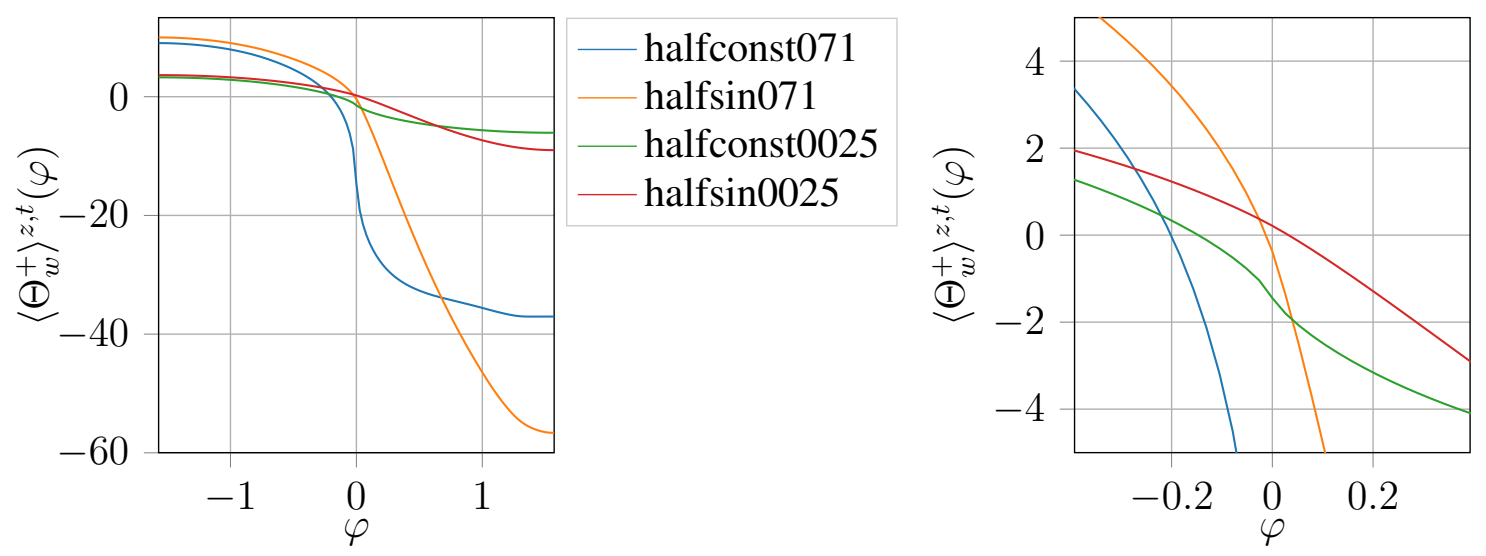

Figure 5: Non-dimensionalized wall temperature $\left\langle\Theta^{+}\right\rangle^{z, t}$ and zoom around $\varphi=0$. 

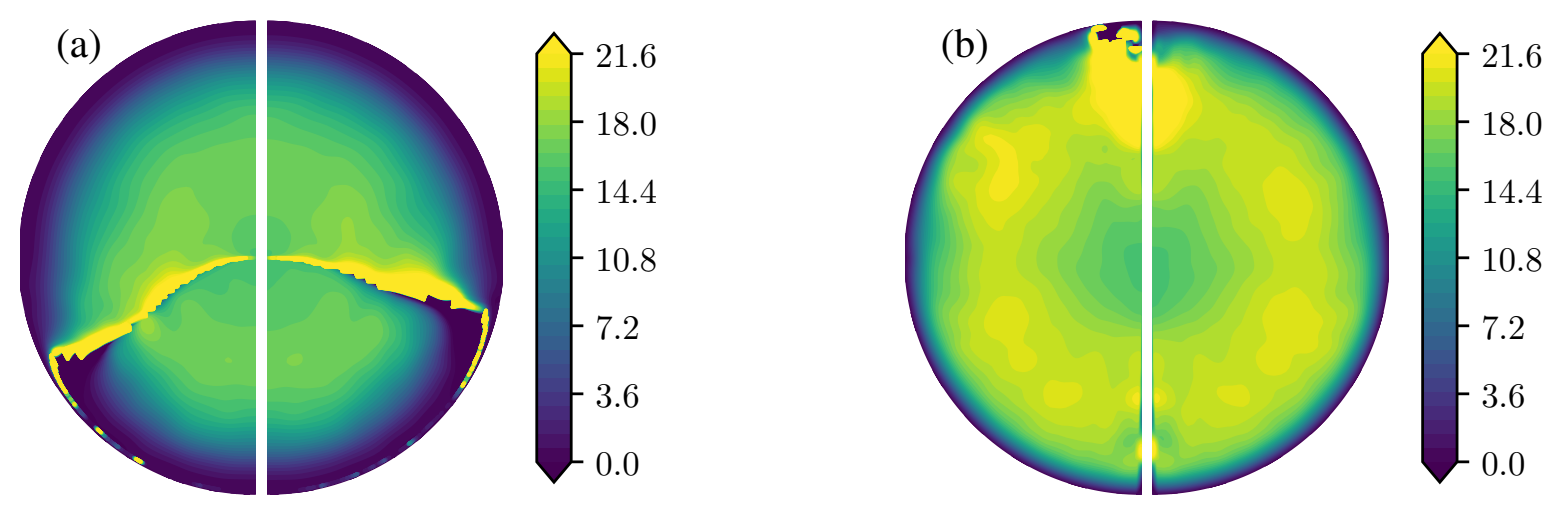

Figure 6: Turbulent thermal diffusivity in (a) radial and (b) azimuthal direction at $\operatorname{Pr}=0.71$ with halfconst on the right and halfsin on the left side of each plot.

Nusselt numbers $\langle N u(\varphi)\rangle$. In the region $0<\varphi<\pi$, where $q_{w}(\varphi) \neq 0$, the magnitude of of $\left\langle\Theta_{w}^{+}\right\rangle^{z, t}$ is largest. For halfsin, both, $q_{w}(\varphi)$ and $-\left\langle\Theta_{w}^{+}\right\rangle^{z, t}$ are increasing from $\varphi=0$ to $\varphi=\pi / 2$, therefore compensating each other and leading to the almost constant $N u(\varphi)$ region observed in Fig. 3. The zoom around $\varphi=0$ explains the singularity of $N u(\varphi)$ for halfsin0025. Only for this setup $\left\langle\Theta_{w}^{+}\right\rangle^{z, t}$ vanishes for positive $\varphi$.

For RANS simulations especially important is the turbulent thermal diffusivity and the turbulent Prandtl number to accurately capture the correct temperature field. They are assessed in the next subsection.

\subsection{Turbulent diffusivity}

The turbulent thermal diffusivity $\alpha_{t}$ in radial and azimuthal direction is shown in Fig. 6 for $\operatorname{Pr}=0.71$. The singularity in $\alpha_{t, r}$ at around $\varphi=-20^{\circ}$ for halfsin 071 arises from its definition

$$
\alpha_{t, r}=-\frac{\left\langle u_{r} \vartheta\right\rangle^{z, t}}{\frac{\partial\langle\Theta\rangle^{z, t}}{\partial r}}, \quad \alpha_{t, \varphi}=-\frac{\left\langle u_{\varphi} \vartheta\right\rangle^{z, t}}{\frac{1}{r} \frac{\partial\langle\Theta\rangle^{z, t}}{\partial \varphi}}, \quad \nu_{t}=-\frac{\left\langle u_{r} u_{z}\right\rangle^{\varphi, z, t}}{\frac{\partial\left\langle U_{z}\right\rangle^{\varphi, z, t}}{\partial r}}
$$

when the temperature gradient in the denominator vanishes (compare with Fig. 4 (a)). Apart from that, $\alpha_{t, r}$ appears to be independent of the azimuthal location, as suggested by Antoranz et al. [5] and Marocco et al. [4]. Similarly, $\alpha_{t, \varphi}$ appears to be independent of $\varphi$ except for the singularity at $\varphi=\pi / 2$. For $\operatorname{Pr}=0.025$ the same trends are observed at lower values of both $\alpha_{t, r}$ and $\alpha_{t \varphi}$.

The turbulent Prandtl number $\operatorname{Pr}_{t}=\nu_{t} / \alpha_{t}$ is shown in Fig. 7 for radial and azimuthal direction. Here, $\alpha_{t, r}$ is averaged over $\pi / 4<\varphi<\pi / 2$ and $\alpha_{t, \varphi}$ over $0<\varphi<\pi / 4$ [5], where no singularity occurs. Clearly, Fig. 7 shows the strong influence of the molecular Prandtl number, resulting in $\left\langle P r_{t, r}\right\rangle^{\varphi, z, t}=\left\langle P r_{t, \varphi}\right\rangle^{\varphi, z, t} \approx 0.7$ and $\left\langle P r_{t, r}\right\rangle^{\varphi, z, t}=\left\langle P r_{t, \varphi}\right\rangle^{\varphi, z, t} \approx 1.6$ in the center of the pipe for molecular $\operatorname{Pr}=0.71 ; 0.025$, respectively. Moreover, the different wall heat flux distributions only show minor effects on the turbulent Prandtl number, with halfsin071 and halfconst071 being the lower curves, collapsing onto each other.

\section{Conclusions}

Direct numerical simulations on azimuthally inhomogeneous thermal boundary conditions are performed and compared to homogeneous reference cases. The global Nusselt number, defined 

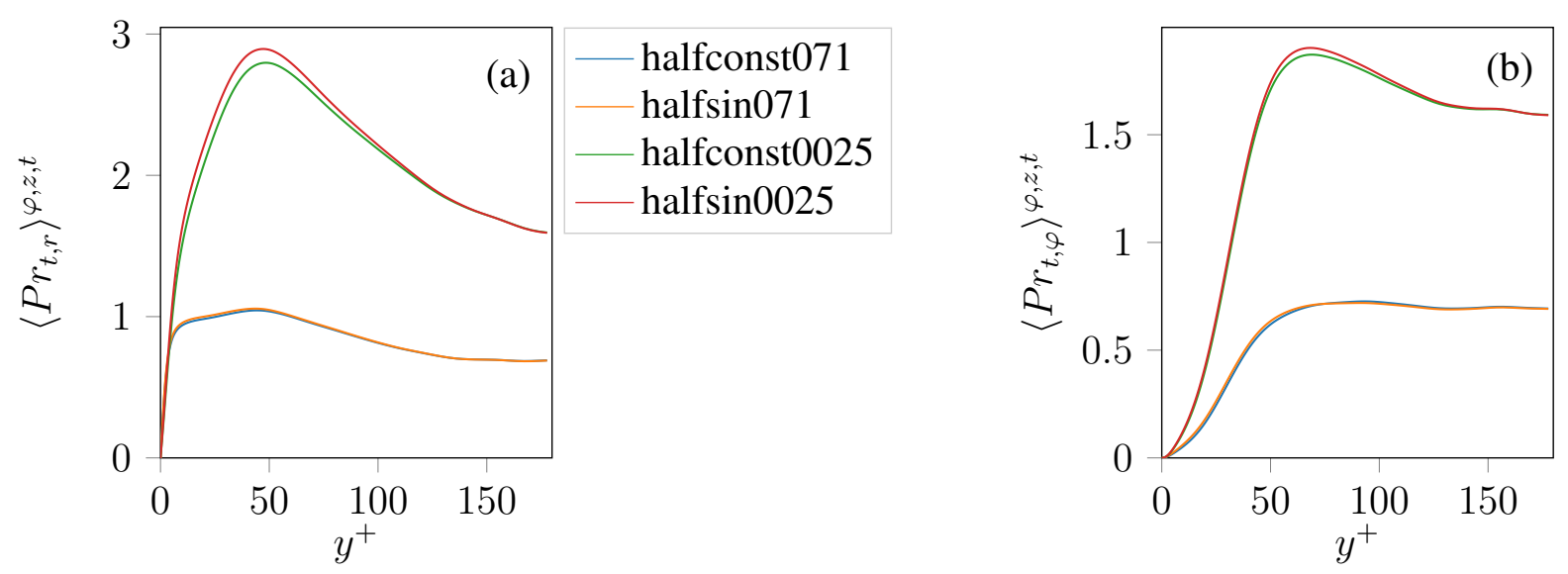

Figure 7: Turbulent Prandtl numbers in (a) radial and (b) azimuthal direction.

by the space and time averaged wall heat flux, appears to be unaffected by the azimuthal inhomogeneity of the prescribed wall heat flux. However, the local Nusselt number, defined by the local wall heat flux, is considerably smaller than the global one except for the transition between adiabatic and heated wall. The distribution of the non-dimensionalized wall temperature and the prescribed wall heat flux compensate each other such that the local Nusselt number is almost constant over a wide range within the heated section of the wall. The turbulent Prandtl number, being anisotropic in the inhomogeneous setup, is mostly affected by the molecular Prandtl number and only to a minor extent by the distribution of wall heat flux.

\section{Acknowledgements}

The simulations were performed on the national supercomputer Cray XC40 at the High Performance Computing Center Stuttgart (HLRS) under the grant number ctbctpf/44106.

\section{References}

[1] J. Pacio et al. "Thermodynamic evaluation of liquid metals as heat transfer fluids in concentrated solar power plants”. In: Applied Thermal Engineering 60 (2013), pp. 295-302.

[2] M. Piller. "Direct numerical simulation of turbulent forced convection in a pipe". In: International Journal for Numerical Methods in Fluids 49.6 (2005), pp. 583-602.

[3] L. Redjem-Saad, M. Ould-Rouiss, and G. Lauriat. "Direct numerical simulation of turbulent heat transfer in pipe flows: Effect of Prandtl number". In: International Journal of Heat and Fluid Flow 28.5 (2007), pp. 847-861.

[4] L. Marocco et al. "Numerical analysis of a solar tower receiver tube operated with liquid metals”. In: International Journal of Thermal Sciences 105 (2016), pp. 22-35.

[5] A. Antoranz et al. "Numerical simulation of heat transfer in a pipe with non-homogeneous thermal boundary conditions". In: International Journal of Heat and Fluid Flow 55 (2015), pp. 45-51.

[6] I. F. Okafor, J. Dirker, and J. P. Meyer. "Influence of circumferential solar heat flux distribution on the heat transfer coefficients of linear Fresnel collector absorber tubes". In: Solar Energy 107 (2014), pp. 381-397. 
[7] A. Antoranz et al. "Extended proper orthogonal decomposition of non-homogeneous thermal fields in a turbulent pipe flow". In: International Journal of Heat and Mass Transfer 118 (2018), pp. 1264-1275.

[8] A. Fritsch et al. "A comparison between transient CFD and FEM simulations of solar central receiver tubes using molten salt and liquid metals". In: Solar Energy 155 (2017), pp. 259-266.

[9] O. Flores et al. "Thermal Stresses Analysis of a Circular Tube in a Central Receiver". In: Energy Procedia 49 (2014), pp. 354-362.

[10] C. Marugán-Cruz et al. "Heat transfer and thermal stresses in a circular tube with a non-uniform heat flux". In: International Journal of Heat and Mass Transfer 96 (2016), pp. 256-266.

[11] W. R. Logie, J. D. Pye, and J. Coventry. "Thermoelastic stress in concentrating solar receiver tubes: A retrospect on stress analysis methodology, and comparison of salt and sodium". In: Solar Energy 160 (2018), pp. 368-379.

[12] NEK5000 v17.0. Release Date 2017/12/17. Argonne National Laboratory, Illinois. Available: https://nek5000.mcs.anl.gov.

[13] A. T. Patera. "A spectral element method for fluid dynamics: Laminar flow in a channel expansion”. In: Journal of Computational Physics 54.3 (1984), pp. 468-488.

[14] S. Saha et al. "The influence of pipe length on thermal statistics computed from DNS of turbulent heat transfer". In: International Journal of Heat and Fluid Flow 32.6 (2011), pp. 1083-1097.

[15] G. K. El Khoury et al. "Direct numerical simulation of turbulent pipe flow at moderately high reynolds numbers". In: Flow, Turbulence and Combustion 91.3 (2013), pp. 475495.

[16] F. P. Incropera et al. Fundamentals of heat and mass transfer. 6. ed. Hoboken, NJ [u.a.]: Wiley, 2007.

[17] V. Gnielinski. "Neue Gleichungen für den Wärme- und den Stoffübergang in turbulent durchströmten Rohren und Kanälen". In: Forschung im Ingenieurwesen A 41.1 (Jan. 1975), pp. 8-16.

[18] J. Pacio, L. Marocco, and T. Wetzel. "Review of data and correlations for turbulent forced convective heat transfer of liquid metals in pipes". In: Heat and Mass Transfer 51.2 (2015), pp. 153-164.

[19] B. Lubarsky and S. J. Kaufman. Review of experimental investigations of liquid-metal heat transfer. Technical Note 3336. Lewis Flight Propulsion Laboratory Cleveland, Ohio: National Advisory Comitee for Aeronoutics, 1955.

[20] E. Skupinski, J. Tortel, and L. Vautrey. "Determination des coefficients de convection d'un alliage sodium-potassium dans un tube circulaire". In: International Journal of Heat and Mass Transfer 8.6 (1965), pp. 937-951. 